\title{
A parametric study of DIC measurement uncertainties on cracked metals
}

\author{
Tim Wigger, Colin Lupton, Jie Tong* \\ Mechanical Behaviour of Materials Laboratory, School of Engineering, University of \\ Portsmouth, Anglesea Building, Anglesea Road, Portsmouth PO1 3DJ, United Kingdom. \\ ${ }^{*}$ Corresponding author
}

\begin{abstract}
Displacements and strains in the presence of a crack in welded steels have been studied using digital image correlation (DIC) under zero load and selected loading conditions. A parametric study has been carried out to assess the effects of some of the key parameters, including subset size, step size and the size of the measurement window, on the measurement uncertainties for systematic and random errors. The assessments were carried out a region around the crack tip, where high strain gradients and inhomogeneous deformation present significant challenges for DIC measurements.
\end{abstract}

\section{Keywords}

Crack; DIC; error analysis; steel; welding

\section{Introduction}

Digital image correlation (DIC) is a non-contact surface measurement method that acquires images of an object, un-deformed and deformed, stores the images in digital form and performs image analyses to extract full-field information on surface deformation of the object. One of the most commonly used approaches of DIC utilises random patterns and compares sub-regions throughout the image to obtain a full-field of measurements. ${ }^{[1]}$ The basic principles of DIC were developed in the 1980's ${ }^{[2,3]}$. Later algorithms for more robust and faster convergence were proposed ${ }^{[4,5]}$, which enabled this technique to be used for a range of applications, including displacement and strain measurements for fracture or crack growth analysis ${ }^{[6]}$. DIC was also utilised for the determination of stress intensity factors ${ }^{[7,8]}$, for fatigue crack growth analysis ${ }^{[9-11]}$ and more recently carried out in situ ${ }^{[12-14]}$, which allows tracking of instantaneous near-tip deformation during crack propagation under cyclic loading.

A variety of errors may arise during the processes of image acquisition and correlation analysis, and they need to be carefully assessed before the DIC method can be used with confidence for in situ data capture and analysis. This is particularly important in cases where the measured strains are sensitive to local events or where large gradients prevail, such as 
areas in the vicinity of a crack tip. A number of parameters may contribute to the measurement uncertainty. For example, it is known that the accuracy and precision of DIC measurements are significantly influenced by the characteristics of the speckle patterns or the material surface texture under study ${ }^{[15-24]}$. Furthermore, systematic errors associated with hardware such as setup and optics ${ }^{[19,25-27]}$, software such as correlation algorithms ${ }^{[15,28]}$ and related sub-pixel registration algorithms together with subset shape functions ${ }^{\text {[28-34] }}$ are also known to significantly affect the measurement quality.

The subset size, a key parameter for image correlation, has been found to be critical for the measurement accuracy [6,35,36]. Much work has been focused on the selection of an "optimum" subset size, either based on displacement analysis ${ }^{[15,17,21,22,37]}$, on strain analysis ${ }^{[16]}$ or both ${ }^{[18]}$. The influence of the step size was discussed in ${ }^{[16]}$ and its effects on the measurements investigated in ${ }^{[37,38]}$, whilst the impact of the applied strain level was studied in ${ }^{[15,37,39]}$. The measurement window (MW), a user-defined area on a displacement/strain map, is usually utilised to obtain average displacements or strains for the region of interest. In this study, however, the effect of the size of the MW on the errors was examined by analysing the data within a MW. The standard deviations for random errors and average systematic errors were obtained from all the data points inside a MW. The impacts of subset size, step size and the size of MW on measurement uncertainties have not yet been studied in cases of cracked specimens under load.

Most of the previous work on error assessments was carried out by examining computergenerated or artificially manipulated speckle patterns. In physical experiments, painted speckles are sized around 5-10 $\mu \mathrm{m}{ }^{[40]}$, and are considered suitable for a resolution of about $10 \mu \mathrm{m} /$ pixel $^{[41,42]}$, though resolutions of 2.5 to $0.7 \mu \mathrm{m} /$ pixel have been achieved recently ${ }^{[12-}$ 14]. In order to achieve a resolution of around $0.5 \mu \mathrm{m} / \mathrm{pixel}$, which is close to the optical microscopy resolution limit of circa $0.2 \mu \mathrm{m}{ }^{[43]}$, methods such as exposing material surface texture by etching have been considered. Etching has been used for steel ${ }^{[44]}$, copper ${ }^{[45]}$ and at micro-scale under SEM ${ }^{[46]}$, where sufficient contrast may be achieved from fine-grained microstructures or materials with sub-grain features, such as in martensite or bainite.

The aim of this work is to provide a systematic study of the influence of subset size, step size and the size of MW on the DIC measurement errors of displacements and strains under load in cracked samples. We aim to establish a baseline error band to give confidence to subsequent fatigue crack growth analyses. Specifically, in situ measurements of displacements and strains in the vicinity of a fatigue crack were carried out on pre-cracked specimens of welded dissimilar steels under selected loads, and the effects of the selected 
parameters on the measured random and systematic errors in displacements and strains were assessed.

\section{Materials and Methods}

The material is a welded $30 \mathrm{Cr} 2 \mathrm{Ni} 4 \mathrm{MoV}$ power plant rotor steel, which was previously examined for its microstructure and mechanical properties ${ }^{[47]}$. The error analysis was performed on three compact tension (CT) specimens designated for a study of fatigue crack growth at a later stage. Fig. 1 shows a typical pre-cracked CT specimen with base metal (BM), weld metal (WM) and heat-affected zone (HAZ) indicated. The HAZ consists of martensites and bainites, with sizes of grains post welding varying between 7 to $32 \mu \mathrm{m}$ and some larger grains up to $50 \mu \mathrm{m}$ close to the welding line due to more intense heat exposure. The weld metal is mainly composed of bainites and $\delta$-ferrites, with an average packet size of $100 \mu \mathrm{m}$. A summary of the microstructural characteristics, chemical composition and mechanical properties of BM, HAZ and WM is presented in Table 1.

All specimens were first polished with $0.5 \mu \mathrm{m}$ silica, then the surface in an area approximately $10 \mathrm{~mm}$ by $10 \mathrm{~mm}$ around the crack tip was etched with a $3 \%$ nitric acidethanol solution for about 30 seconds. This procedure revealed surface microstructural features (Fig. 3a) with an average feature size of about $5 \mu \mathrm{m}$.

\subsection{Displacement and strain determination}

The software DaVis (version 8.4, LaVision $\mathrm{GmbH}$, Göttingen, Germany) was used in the image correlation procedure for the error analysis. A zero-normalised sum of squared differences algorithm ${ }^{[48]}$ with Lucas Kanade approach ${ }^{[49,50]}$ and a sixth order spline interpolation scheme for sub-pixel accuracy were employed to obtain the displacement vector fields. An explicit quantitative measure of correlation quality in terms of a correlation coefficient is not provided by the algorithm, although poor correlations are indicated. Based on the displacement fields, strain maps $\left(\varepsilon_{x x}, \varepsilon_{y y}, \varepsilon_{x y}\right)$ were generated. The strain component for a given data point at position $x, y$ is calculated using the neighbouring displacement data points at positions $x-1, x+1$ and $y-1, y+1$, without filtering. A first order shape function was used for all image correlations.

\subsection{Experimental setup}

The error analyses were carried out under three selected loading conditions equivalent to stress intensity factors (SIF) of $0,20,30 \mathrm{MPa} \sqrt{\mathrm{m}}$ using an Instron servo-hydraulic testing machine. A LaVision DIC system was used for image acquisition, including a 12-bit CCD camera mounted on an optical microscope with a Schneider Kreuznach $50 \mathrm{~mm}$ lens and an extension tube of $100 \mathrm{~mm}^{[14]}$ (Fig. 2). The lighting setup and the aperture position were 
critical for imaging the etched surface, since the surface reflection of the metal is less bright and shows less contrast than e.g. a speckle pattern on painted white background. Two LED flash lights were utilised for this purpose to obtain good quality images. All images were taken with $2456 \times 2058$ pixels on a field of view of $1330 \times 1120 \mu \mathrm{m}^{2}$, resulting in a spatial resolution of $0.54 \mu \mathrm{m} /$ pixel.

Six images of each specimen were taken under zero load using identical imaging parameters. Image correlation was performed on each with the first image used as the reference image, within a correlation region of interest (ROI) of approximately $1300 \times 1100$ $\mu \mathrm{m}^{2}\left(2410 \times 2040\right.$ pixels $\left.^{2}\right)$. Similarly, five images were acquired per specimen while applying constant loads corresponding to SIF of $20 \mathrm{MPa} \sqrt{\mathrm{m}}$ and $30 \mathrm{MPa} \sqrt{\mathrm{m}}$ to open the crack. These images were compensated for rigid body motion and correlated with a zero-load reference image, resulting in a smaller ROI of around $1250 \times 900 \mu \mathrm{m}^{2}\left(2320 \times 1670\right.$ pixels $\left.^{2}\right)$.

Five displacement and strain maps were generated per specimen, and three specimens were measured in total, thus fifteen images $(n=15)$ were examined for each of the selected parameters. Only y-components (perpendicular to the crack plane) in the measured displacements and strains are presented in the error analysis.

The effects of the selected parameters on both random errors (noise) and systematic errors (bias) were assessed. For the evaluation of subset size and step size, the data points inside a measurement window (MW) of $1390 \times 1000$ pixels $^{2}\left(750 \times 540 \mu \mathrm{m}^{2}\right)$ surrounding the crack tip were analysed for each displacement and strain map. Subsequently, the effects of the MW on the measurement uncertainties were also studied by analysing the data from selected sizes of MW centred on the crack tip, with the subset and step sizes fixed.

The sources of errors include systematic and random types. Systematic errors may be introduced by mismatched shape functions; while random errors are essentially noise ${ }^{[18,30]}$. For each parameter, the random errors were investigated first, using the standard deviations (SD) of the displacement and strain data points inside the given MW. Since the actual deformation is not known in our experiments, the systematic displacement errors were assessed based on an approximation procedure proposed by $\mathrm{Xu}$ et al. ${ }^{[37]}$. For the $\mathrm{y}$ component:

$$
S_{v}^{\text {approx }}(x, y)=\frac{M(M+1)}{6} \Delta v(x, y)
$$

Where $\Delta v$ is the second order displacement gradient in y direction, $\Delta$ is the Laplace operator and $M=$ (subset size -1$) / 2$. An estimate for $\Delta v$ may be obtained by comparing two displacement maps generated using a pair of subset sizes: 


$$
\Delta v(x, y) \approx \frac{6}{M_{1}\left(M_{1}+1\right)-M_{2}\left(M_{2}+1\right)}\left(v_{M_{1}}^{D I C}(x, y)-v_{M_{2}}^{D I C}(x, y)\right)
$$

Where $M_{1}$ and $M_{2}$ are the pair of subsets chosen for the comparison.

The convergence and the effectiveness of the estimates from Eq. 2 were examined using a synthetic dataset (DIC challenge sample $14 L 5^{[51]}$ ). For a suitable choice of a subset pair for the $2^{\text {nd }}$ order gradient estimation, the known $2^{\text {nd }}$ order displacement gradients $\Delta v$ were compared with the estimated gradients, obtained by Eq. 2, using selected subset size combinations. The "best fit" was achieved using a subset pair of $M_{1}=75$ pixels and $M_{2}=35$ pixels (Fig. 4a), although slightly higher relative errors occurred in areas of low gradients. The resulting gradients were consequently used for the bias error approximation $S_{v}(E q .1)$ on sample $14 \mathrm{~L} 5^{[51]}$, which was then compared with the actual bias error. The actual bias error was obtained from the difference between the known displacements and those calculated by DIC using the selected subset sizes ( $M=35,49,75$ pixels). A favourable comparison is obtained between the actual (solid) and estimated (dashed) bias errors, as shown in Fig. 4b, with a difference no more than $20 \%$ mostly, with occasional exceptions in areas of low deformation. Note that the choice of the subset pair $\left(M_{1}=75\right.$ pixels and $M_{2}=35$ pixels) may be specific to the artificial data tested, whilst the characteristics of a "real" deformation field may well be different hence the present results should not be generalised.

A virtual strain gauge (VSG) study ${ }^{[52]}$ was performed to assess the systematic strain errors. This method places a VSG close to the crack tip and assesses the spatial resolution of the measurements and the level of noise (SD) towards an "optimum" solution. The parameter sets that offer a good "convergence" with low SDs are recommended for use. In this work, several positions ahead and behind the crack tip were evaluated for a suitable position of the placement of VSG, and the strongest signals were found to be at the crack tip, where the VSG was consequently placed. The strains were calculated using a strain calculation window, SW ${ }^{[52]}$, which contains an array of $3 \times 3$ immediately adjacent neighbouring points. The normal strains were evaluated using combinations of six selected subset sizes $(23,35$, $43,49,61,75$ pixels) and five selected step sizes (4, 6, 9, 12, 19 pixels). The subset and step sizes which produce the most converged results were subsequently identified.

\section{Results}

\subsection{The effects of subset size}

The standard deviations (SDs) of displacement and strain in y-direction under zero load are shown in Fig. 5 as a function of subset size, which was varied in five steps $(23 \times 23,35 \times 35$, $49 \times 49,75 \times 75,147 \times 147$ pixels $^{2}$ ), whilst the step size was fixed to 6 pixels. The random 
errors, also referred to as noise floor, in both displacement and strain decreased drastically with the increase of subset size and appear to stabilise when the subset size is $50 \times 50$ pixels ${ }^{2}$ or above.

Further studies were carried out under loads, at stress intensity factors (SIF) of 20 and 30 $\mathrm{MPa} \sqrt{\mathrm{m}}$. Random and systematic errors were estimated similarly to those determined at zero load.

Fig. 6 shows some of the full-field distributions of random and systematic displacement errors in the ROI for the loaded case $\mathrm{K}=20 \mathrm{MPa} \sqrt{\mathrm{m}}$, for three selected subset sizes and a step size of 6 pixels. The levels of the errors are shown in the scaling bars below, assuming that a SD of less than 0.5 pixel indicates sufficient matching. For random errors (left column), a large number of erroneous regions is present for the smallest subset of $23 \times 23$ pixels $^{2}$ (Fig. 6a) due to poor correlation; whilst the errors decrease in number and intensity with the increase in subset size (Fig. 6b, c), with the lowest level of errors found in the largest subset. By contrast, for systematic errors (right column), the smallest subset (23x23 pixels ${ }^{2}$, Fig. 6d) seems to return the lowest level of errors compared with the medium-sized subset $(49 \times 49$ pixels $^{2}$, Fig. 6e) and largest subset $\left(75 \times 75\right.$ pixels $^{2}$, Fig. $\left.6 f\right)$. The crack is clearly identifiable by enhanced errors in all, but particularly in the middle and large subset cases; whilst errors induced by noise are found in low deformation areas (Fig. 6f).

The quantitative results are presented in Fig. 7. For the random errors, the measured standard deviation of the displacement seems to be insensitive to the subset size but sensitive to the load level (Fig. 7a); whilst the noise in the measured strain seems to decrease with the increase of subset size (Fig. 7b), stabilising at a subset size of about $50 \times 50$ pixels $^{2}$. On the other hand, the displacement bias (Fig. 7c) increases for both loaded cases, most significantly at large subset sizes. At zero load, a low level of systematic errors was found, estimated from Eq. 1 and Eq. 2, using the experimental data. This is possibly due to noise induced by test rig vibration as no bias was found using synthetic data.

\subsection{The effects of step size}

Selected step sizes $(2,4,6,9,12,19$ pixels) were examined to assess their effects on the measurement uncertainties with a fixed subset size $\left(49 \times 49\right.$ pixels $\left.{ }^{2}\right)$. Fig. 8 shows the effects of step size on random ( $a, b)$ and systematic (c) displacement errors. The number of data points is also indicated as well as the step size.

For random errors, whilst the SDs in the measured displacements seem to be generally unaffected by the step size (Fig. 8a), a small step size or more data points appear to increase the SD in the measured strains, particularly under loads (Fig. 8b). The systematic 
displacement error also shows a trend of decrease with the increase of step size or lower number of data points (Fig. 8c).

\subsection{Systematic strain errors}

Using a VSG, the strains at the points along the line perpendicular to the global crack plane and centred at the crack tip (Fig. 9a) were analysed. The standard deviations of the normal strains along the line were calculated under zero load to determine the base-line first, followed by the strain values obtained under loads for the selected parameter sets (Fig. 9b, c). The results from all combinations of subset size and step size are presented, with four most promising data sets highlighted (details given in Table 2).

\subsection{The effects of the size of the measurement window}

The influences of the size of the measurement window (MW) on the measurement uncertainties are shown in Fig. 10, as a function of the size of MW (selected as $12 \times 12$, $18 \times 18,36 \times 36,60 \times 60,93 \times 93,130 \times 130,186 \times 186$ pixels $^{2}$ ) as well as the number of data points inside the MW. The subset size was kept constant at $49 \times 49$ pixels $^{2}$ and the step size at 6 pixels. Each measurement window was centred around the crack tip. The random errors in both displacement and strain seem to increase with the increase of the size of MW (or the number of data points) and load level, similar to the random errors in the measured strains with respect to step size (Fig. 8b).

The systematic displacement errors (Fig. 10c) appear to decrease with the increase of the size of measurement window (or data points), and the trend seems to be more pronounced at higher load $(\mathrm{K}=30 \mathrm{MPa} \sqrt{\mathrm{m}})$.

\section{Discussion}

A systematic study of DIC measurement uncertainties in cracked specimens of metals has been carried out with and without loads, for the purpose of near-tip strain mapping of fatigue cracks. The effects of some of the key DIC parameters on the measurement errors have been examined. Specifically, the influence of subset size, step size and size of the measurement window have been examined. There are complex sources of errors behind and ahead of the crack tip, as low-quality correlation due to the discontinuity between the crack flanks leads to a high level of noise and deformation gradients, hence locally high random errors at small subsets; whilst large and complex deformations introduce high bias at large subsets.

\section{Subset size}


Random strain errors are reduced with the increase of subset size, due to higher confidence gained in image correlation where a greater number of features are available in larger subset sizes ${ }^{[15-17,30]}$, leading to a decrease in the standard deviations. The trends under zero load shown in Fig. 5 are consistent with the published work ${ }^{[16,17]}$, although most of the previous experiments were conducted ex situ with very fine, artificial speckle patterns generated by algorithms. In this study, physical features were used in physical experiments, where the noise level was found to stabilise when the subset size is $50 \times 50$ pixels $^{2}$ or above, a value used in a previous study of full-field near-tip strain mapping ${ }^{[14]}$. Under loads, large deformations and large gradients as well as discontinuities due to the crack opening present major challenges to image correlation. As shown in Fig. $7 \mathrm{~b}$, increasing load led to significantly increased SDs, although the trend in strain random errors under loads is similar to that under zero load, i.e. higher SDs for smaller subset sizes. For random displacement errors, the effects of the subset size are completely overshadowed by the influence of the load level (Fig. 7a). The systematic displacement errors were estimated using Eq. 1 and Eq. 2, which are subject to the sizes of the chosen pair of subsets in Eq. 2, which may affect the estimated results (Fig. 4).

Random error distributions of displacement may be appreciated in full-field SD images obtained under load $(\mathrm{K}=20 \mathrm{MPa} \sqrt{\mathrm{m}})$ presented in Fig. 6a-c. At a small subset of $23 \times 23$ pixels $^{2}$ (Fig. 6a), high levels of errors (above 0.5 pixels) are distributed widely as small subsets do not contain sufficient information for matching low-contrast areas or discontinuities. On the other hand, at a larger subset size $\left(147 \times 147\right.$ pixels $^{2}$ Fig $\left.6 \mathrm{c}\right)$, the errors appear to be reduced and are confined to the cracked area. In the latter case the deformation is averaged over a large area with an overlap of $96 \%$ (step size 6 pixels), thus essentially similar information is used to calculate the neighbouring vectors ${ }^{[38]}$. In Fig 7, the random SD in the measured displacements increases almost linearly with load from around 4 pixels for $\mathrm{K}=20 \mathrm{MPa} \sqrt{\mathrm{m}}$ to 6 pixels for $\mathrm{K}=30 \mathrm{MPa} \sqrt{\mathrm{m}}$. Similarly, the strain errors are $2.5 \%$ for $\mathrm{K}=20 \mathrm{MPa} \sqrt{\mathrm{m}}$ and $3.8 \%$ for $\mathrm{K}=30 \mathrm{MPa} \sqrt{\mathrm{m}}$ at a subset size of $49 \times 49$ pixels ${ }^{2}$.

There seems to be no effect of the load level on the systematic displacement errors (Fig. $7 c)$, as the displacement errors increase with the subset size for both load cases. The fullfield results for systematic errors at $\mathrm{K}=20 \mathrm{MPa} \vee \mathrm{m}$ (Fig. 6d-f) show the impact of subset size clearly. Significantly higher and widely spread errors were found in large subsets $(>50 \times 50$ pixels $^{2}$; Fig 6f). This is probably due to the large complex deformation and high gradients around the crack discontinuity, leading to undermatched subset shape functions ${ }^{[15,28,30,37]}$. Slightly higher bias errors may be obtained in low deformation areas from the estimation method, although this has negligible impact on the assessment of deformation in the vicinity of a crack, which is always high. 
The ratio between the average feature size (about 10 pixels) and the recommended subset size is about 0.2 in this study. This compares favourably with estimated ratios of 0.1 to 0.3 reported in the literature ${ }^{[15-18]}$. It seems that a subset size about 5 times of the speckle/feature size would suffice in DIC measurements. It has to be remarked that the speckle size is only one of many parameters used to characterise speckle patterns. Other parameters such as morphology ${ }^{[22]}$, intensity gradients ${ }^{[17]}$ and entropies ${ }^{[18,20]}$ might be subjects of further studies. Overall, considering both random and systematic errors, a compromise may be found at a subset size about $50 \times 50$ pixels $^{2}$, which is approximately five times the surface feature size.

Step size

Although the effect of step size on the random displacement errors seems very small (Fig. $8 a$ ), a decrease in step size (or increase in the number of data points) increases the strain SD under load (Fig. 8b). This is possibly due to the enhanced overlapping at small steps when more data points are available, also increased spatial resolution where variation in the resulting maps becomes more evident. At large step sizes, fewer data points are available and spatial resolution decreases, the resulting less complex displacement field might be described with reduced bias (Fig. 8c).

Despite the increased errors, smaller step sizes do provide much higher spatial resolution. A ratio of step size over subset size of around 1/4 (12 pixels for a 49-pixel subset) is usually recommended, which appears to provide reasonably low bias for the cases considered here.

\section{Systematic strain errors}

The virtual strain gauge approach appears to be appropriate in producing satisfactory estimates for the impact of selected parameters on the strain measurement bias, the latter could not otherwise be assessed by applying the method of Xu et al. ${ }^{[37]}$ to the experimental data. Smaller subsets (23 or 35 pixels $^{2}$ ) give rise to large variations; whilst larger subsets (61 or 75 pixels $^{2}$ ) tend to underestimate the strain by $50 \%$ or more. An "optimum" was found to be between subsets $43 \times 43$ and $49 \times 49$ pixels $^{2}$, which is consistent with our assessments of random and systematic errors (Fig.6; Fig. 7). The best choice for the step size seems to be in the range of 9 to 12 pixels, which approximately corresponds to one quarter of the subset size.

\section{Measurement window}

The random errors in both displacement and strain increase with the size of MW (or the number of data points). Low levels of SD are obtained at with small MWs due to less variation when fewer data points are considered in a small MW. A large MW may contain a 
mixture of high deformation regions near the crack and low deformation in the surrounding areas, hence a high SD; whilst a small MW may contain only a high or low deformation region, hence less spread of data and a low SD.

Contrary to the above trend, the systematic displacement errors decrease significantly with the increase of the size of MW where more data points are available (Fig. 10c). Only high gradients may occur in small MWs with few data points located near the crack tip, which are difficult to match using a low order subset shape function hence higher bias is measured; whereas a large MW may contain a mixture of low and high gradients, and the average estimated error may appear to be lower. The fidelity of the measurements is also a key issue in the choice of MW. A compromise should be sought between achieving a desirable spatial resolution and an acceptable level of measurement errors.

The influence of material variation on the measurement uncertainties appears to be small. Although some of the images were captured on the specimen surface with the heat affected base metal on the top half and the weld metal on the bottom half, the measured errors do not appear to be related to local material characteristics or microstructure details such as grain size.

\section{Limitations}

We have considered a case with a crack introduced in the centre of the ROI. The influence of crack opening in the loaded cases, as a discontinuity, on the image correlation and error assessment should be taken into account in the interpretation of the results. In applications, the crack flank areas should be masked to improve the correlation quality and the fidelity of the measurements. Our results provide a worst-case scenario where the discontinuity was included in the assessments, hence caution should be exercised when reading the results.

The estimation of the systematic errors was based on analytical solutions ${ }^{[37]}$ that depend on displacement gradients, which can change with the size of the subset pair used for their estimations. Also, the VSG approach for the strain bias assessment utilises only a onedimensional "virtual gauge" in a 2D strain field, hence its limitation should be taken into account. The study is further limited in that it was carried out using a commercial software (LaVision DaVis 8.4) which does not provide an explicit measure of correlation quality.

Nevertheless, we hope the results are useful in shedding some light on the DIC measurement uncertainties of cracked bodies under load for further applications of DIC in engineering structures and components. 


\section{Conclusions}

An error assessment of displacement and strain obtained by in situ digital image correlation experiments has been carried out. The effects of selected key parameters, including subset size, step size and the size of the measurement window, on the standard deviations and systematic errors of displacement and strain have been examined with and without the load in cracked dissimilar metals. An "optimal" subset size was found to be about 5 times the speckle/feature size as a result of the trade-off between systematic and random errors. A step size of $1 / 4$ of the subset size or smaller is recommended. Small measurement windows tend to produce results with low noise, although they are perhaps more biased and locationdependent under load. Under the selected experimental conditions examined, random errors appear to be predominant.

\section{Acknowledgements:}

The authors are grateful to Dr M-L Zhu of East China University of Science and Technology, China, for providing the specimens and helpful discussion; and to Mr D-Q Wang, also of East China University of Science and Technology, China, for fatigue pre-cracking.

\section{References}

[1] H. Schreier, J. Orteu, M.A. Sutton, Image Correlation for Shape, Motion and Deformation Measurements, 1st ed., Springer US, Boston, MA, 2009. doi:10.1007/978-0-387-78747-3.

[2] W.H. Peters, W.F. Ranson, Digital imaging techniques in experimental stress analysis, Opt. Eng. 21 (1982) 427-431. doi:10.1117/12.7972925.

[3] M. Sutton, W. Wolters, W. Peters, W. Ranson, S. McNeill, Determination of displacements using an improved digital correlation method, Image Vis. Comput. 1 (1983) 133-139. doi:10.1016/0262-8856(83)90064-1.

[4] H. Bruck, S. McNeill, M. Sutton, Digital image correlation using Newton-Raphson method of partial differential correction, Exp. Mech. 29 (1989) 261-267. http://www.springerlink.com/index/PL1R80WX70G50055.pdf.

[5] G. Vendroux, W.G. Knauss, Submicron deformation field measurements: Part 2. Improved digital image correlation, Exp. Mech. 38 (1998) 86-92. doi:10.1007/BF02321649.

[6] J. Abanto-Bueno, J. Lambros, Investigation of crack growth in functionally graded 
materials using digital image correlation, Eng. Fract. Mech. 69 (2002) 1695-1711. doi:10.1016/S0013-7944(02)00058-9.

[7] S. Roux, J. Réthoré, F. Hild, Digital image correlation and fracture: an advanced technique for estimating stress intensity factors of 2D and 3D cracks, J. Phys. D. Appl. Phys. 42 (2009) 214004. doi:10.1088/0022-3727/42/21/214004.

[8] J.R. Yates, M. Zanganeh, Y.H. Tai, Quantifying crack tip displacement fields with DIC, Eng. Fract. Mech. 77 (2010) 2063-2076.

[9] J.D. Carroll, W. Abuzaid, J. Lambros, H. Sehitoglu, High resolution digital image correlation measurements of strain accumulation in fatigue crack growth, Int. J. Fatigue. 57 (2013) 140-150. doi:10.1016/j.ijfatigue.2012.06.010.

[10] S. Vanlanduit, J. Vanherzeele, R. Longo, P. Guillaume, A digital image correlation method for fatigue test experiments, Opt. Lasers Eng. 47 (2009) 371-378. doi:10.1016/j.optlaseng.2008.03.016.

[11] F. Mathieu, F. Hild, S. Roux, Identification of a crack propagation law by digital image correlation, Int. J. Fatigue. 36 (2012) 146-154. doi:10.1016/j.jfatigue.2011.08.004.

[12] J. Tong, B. Lin, Y.W. Lu, K. Madi, Y.H. Tai, J.R. Yates, V. Doquet, Near-tip strain evolution under cyclic loading: In situ experimental observation and numerical modelling, Int. J. Fatigue. 71 (2015) 45-52. doi:10.1016/j.ijfatigue.2014.02.013.

[13] Y.-W. Lu, C. Lupton, M.-L. Zhu, J. Tong, In Situ Experimental Study of Near-Tip Strain Evolution of Fatigue Cracks, Exp. Mech. 55 (2015) 1175-1185. doi:10.1007/s11340015-0014-4.

[14] M.L. Zhu, Y.W. Lu, C. Lupton, J. Tong, In situ near-tip normal strain evolution of a growing fatigue crack, Fatigue Fract. Eng. Mater. Struct. (2016) 1-6. doi:10.1111/ffe.12391.

[15] M. Bornert, F. Bremand, P. Doumalin, J.C. Dupre, M. Fazzini, M. Grediac, F. Hild, S. Mistou, J. Molimard, J.J. Orteu, L. Robert, Y. Surrel, P. Vacher, B. Wattrisse, Assessment of digital image correlation measurement errors: Methodology and results, Exp. Mech. 49 (2009) 353-370. doi:10.1007/s11340-008-9204-7.

[16] H. Haddadi, S. Belhabib, Use of rigid-body motion for the investigation and estimation of the measurement errors related to digital image correlation technique, Opt. Lasers Eng. 46 (2008) 185-196. doi:10.1016/j.optlaseng.2007.05.008.

[17] B. Pan, H. Xie, Z. Wang, K. Qian, Z. Wang, Study on subset size selection in digital image correlation for speckle patterns., Opt. Express. 16 (2008) 7037-7048. doi:10.1364/OE.16.007037. 
[18] S. Yaofeng, J.H.L. Pang, Study of optimal subset size in digital image correlation of speckle pattern images, Opt. Lasers Eng. 45 (2007) 967-974. doi:10.1016/j.optlaseng.2007.01.012.

[19] Y. Barranger, P. Doumalin, J.C. Dupré, a. Germaneau, Digital Image Correlation accuracy: influence of kind of speckle and recording setup, EPJ Web Conf. 6 (2010) 31002. doi:10.1051/epjconf/20100631002.

[20] G. Crammond, S.W. Boyd, J.M. Dulieu-Barton, Speckle pattern quality assessment for digital image correlation, Opt. Lasers Eng. 51 (2013) 1368-1378. doi:10.1016/j.optlaseng.2013.03.014.

[21] M. Fazzini, S. Mistou, O. Dalverny, L. Robert, Study of image characteristics on digital image correlation error assessment, Opt. Lasers Eng. 48 (2010) 335-339. doi:10.1016/j.optlaseng.2009.10.012.

[22] D. Lecompte, A. Smits, S. Bossuyt, H. Sol, J. Vantomme, D. Van Hemelrijck, A.M. Habraken, Quality assessment of speckle patterns for digital image correlation, Opt. Lasers Eng. 44 (2006) 1132-1145. doi:10.1016/j.optlaseng.2005.10.004.

[23] P.-C. Hung, a. S. Voloshin, In-plane strain measurement by digital image correlation, J. Brazilian Soc. Mech. Sci. Eng. 25 (2003) 215-221. doi:10.1590/S167858782003000300001.

[24] Z. Sun, J.S. Lyons, S.R. McNeill, Measuring Microscopic Deformations with Digital Image Correlation, Opt. Lasers Eng. 27 (1997) 409-428. doi:10.1016/S01438166(96)00041-3.

[25] D. Zhang, M. Luo, D.D. Arola, Displacement/strain measurements using an optical microscope and digital image correlation, Opt. Eng. 45 (2006) 33605. doi:10.1117/1.2182108.

[26] H.W. Schreier, Advances in Light Microscope Stereo Vision, Exp. Mech. 44 (2004) 278-288. doi:10.1177/0014485104041546.

[27] S. Yoneyama, H. Kikuta, A. Kitagawa, K. Kitamura, Lens distortion correction for digital image correlation by measuring rigid body displacement, Opt. Eng. 45 (2006) 23602. doi:10.1117/1.2168411.

[28] P. Lava, S. Cooreman, S. Coppieters, M. De Strycker, D. Debruyne, Assessment of measuring errors in DIC using deformation fields generated by plastic FEA, Opt. Lasers Eng. 47 (2009) 747-753. doi:10.1016/j.optlaseng.2009.03.007.

[29] H.W. Schreier, J.R. Braasch, M.A. Sutton, Systematic errors in digital image correlation caused by intensity interpolation, Opt. Eng. 39 (2000) 2915. 
doi:10.1117/1.1314593.

[30] H. Schreier, M. a. Sutton, Systematic Errors in Digital Image Correlation Due to Undermatched Subset Shape Functions, Exp. Mech. 42 (2002) 303-310. doi:10.1177/001448502321548391.

[31] P. Bing, X. Hui-min, X. Bo-qin, D. Fu-long, Performance of sub-pixel registration algorithms in digital image correlation, Meas. Sci. Technol. 17 (2006) 1615-1621. doi:10.1088/0957-0233/17/6/045.

[32] H. Shen, P. Zhang, X. Shen, Bias Error Reduction of Digital Image Correlation Based on Kernel, 81 (2015) 16-20. doi:10.14257/astl.2015.81.04.

[33] D. Wang, Y. Jiang, W. Wang, Y. Wang, Bias reduction in sub-pixel image registration based on the anti-symmetric feature, Meas. Sci. Technol. 27 (2016) 35206. doi:10.1088/0957-0233/27/3/035206.

[34] H. Lu, P.D. Cary, Deformation measurements by digital image correlation: Implementation of a second-order displacement gradient, Exp. Mech. 40 (2000) 393400. doi:10.1007/BF02326485.

[35] W.G. Knauss, I. Chasiotis, Y. Huang, Mechanical measurements at the micron and nanometer scales, Mech. Mater. 35 (2003) 217-231. doi:10.1016/S01676636(02)00271-5.

[36] M. Sutton, C. Mingqi, W. Peters, Y. Chao, S. McNeill, Application of an optimized digital correlation method to planar deformation analysis, Image Vis. Comput. 4 (1986) 143-150. doi:10.1016/0262-8856(86)90057-0.

[37] X. Xu, Y. Su, Q. Zhang, Theoretical estimation of systematic errors in local deformation measurements using digital image correlation, Opt. Lasers Eng. 88 (2016) 265-279. doi:10.1016/j.optlaseng.2016.08.016.

[38] Y. Wang, P. Lava, P. Reu, D. Debruyne, Theoretical Analysis on the Measurement Errors of Local 2D DIC: Part II Assessment of Strain Errors of the Local Smoothing Method-Approaching an Answer to the Overlap Question, Strain. (2016) 110-128. doi:10.1111/str.12174.

[39] X. Xu, Y. Su, Y. Cai, T. Cheng, Q. Zhang, Effects of Various Shape Functions and Subset Size in Local Deformation Measurements Using DIC, Exp. Mech. 55 (2015) 1575-1590. doi:10.1007/s11340-015-0054-9.

[40] G. Lionello, L. Cristofolini, A practical approach to optimizing the preparation of speckle patterns for digital-image correlation, Meas. Sci. Technol. 25 (2014) 107001. doi:10.1088/0957-0233/25/10/107001. 
[41] T.A. Berfield, J.K. Patel, R.G. Shimmin, P. V. Braun, J. Lambros, N.R. Sottos, Microand nanoscale deformation measurement of surface and internal planes via digital image correlation, Exp. Mech. 47 (2007) 51-62. doi:10.1007/s11340-006-0531-2.

[42] H. Wang, H. Xie, Y. Li, J. Zhu, Fabrication of micro-scale speckle pattern and its applications for deformation measurement, Meas. Sci. Technol. 23 (2012) 35402. doi:10.1088/0957-0233/23/3/035402.

[43] Rayleigh, XXXI. Investigations in optics, with special reference to the spectroscope, Philos. Mag. Ser. 5.8 (1879) 261-274. doi:10.1080/14786447908639684.

[44] A. El Bartali, V. Aubin, S. Degallaix, Fatigue damage analysis in a duplex stainless steel by digital image correlation technique, Fatigue Fract. Eng. Mater. Struct. 31 (2008) 137-151. doi:10.1111/j.1460-2695.2007.01207.x.

[45] F.M. Sanchez-Arevalo, G. Pulos, Use of digital image correlation to determine the mechanical behavior of materials, Mater. Charact. 59 (2008) 1572-1579. doi:10.1016/j.matchar.2008.02.002.

[46] A. Tatschl, O. Kolednik, On the experimental characterization of crystal plasticity in polycrystals, Mater. Sci. Eng. A. 342 (2003) 152-168. doi:10.1016/S09215093(02)00278-2.

[47] Y.-N. Du, M.-L. Zhu, X. Liu, F.-Z. Xuan, Effect of long-term aging on near-threshold fatigue crack growth of Ni-Cr-Mo-V steel welds, J. Mater. Process. Technol. 226 (2015) 228-237. doi:10.1016/j.jmatprotec.2015.07.023.

[48] D. Fleet, Y. Weiss, Optical flow estimation, Handb. Math. Model. Comput. Vis. (2006) 237-257. doi:10.1007/0-387-28831-7_15.

[49] J.-Y. Bouguet, Pyramidal implementation of the affine lucas kanade feature trackerdescription of the algorithm, Pages.SIc.Edu. 2 (2001) 3. doi:10.1.1.185.585.

[50] B.D. Lucas, T. Kanade, An Iterative Image Registration Technique with an Application to Stereo Vision, Imaging. 130 (1981) 674-679. doi:10.1109/HPDC.2004.1323531.

[51] Society for Experimental Mechanics, Bethel, USA: sem.org/dic-challenge.

[52] P. Reu, Virtual Strain Gage Size Study, Experimental Techniques 39 (2015) 1-3. doi: 10.1111/ext.12172. 


\begin{tabular}{|l|c|c|c|c|c|c|c|c|c|c|c|c|}
\hline & $\mathrm{C}$ & $\mathrm{Cr}$ & $\mathrm{Ni}$ & $\mathrm{Mo}$ & $\mathrm{V}$ & $\mathrm{Mn}$ & $\mathrm{Si}$ & $\mathrm{Cu}$ & $\sigma_{\text {Ys }}$ & $\sigma_{\text {UTS }}$ & $\mathrm{E}$ & Grain size \\
\hline Alloy & wt.\% & wt.\% & wt.\% & wt.\% & wt.\% & wt. $\%$ & wt.\% & wt.\% & $\mathrm{MPa}$ & $\mathrm{MPa}$ & $\mathrm{GPa}$ & $\mu \mathrm{m}$ \\
\hline BM, HAZ & $\leq 0.35$ & $1.5-2.0$ & $3.25-3.75$ & $0.25-0.6$ & $0.07-0.15$ & $0.2-0.4$ & $\leq 0.1$ & $\leq 0.15$ & 814.3 & 910.6 & 217.7 & $7-32$ \\
\hline WM & 0.19 & 0.75 & 2.35 & 0.71 & & 1.54 & 0.42 & & 772.4 & 833.9 & 210.4 & 100 \\
\hline
\end{tabular}

Table 1. A summary of chemical composition, mechanical properties and microstructural characteristics of the constituents in the welded specimen.

\begin{tabular}{|l|rrrr|rrrr|}
\hline Stress intensity factor & \multicolumn{4}{|c|}{20 MPaVm } & \multicolumn{4}{c|}{30 MPaVm } \\
\hline Subset size (SS) & 35 & 43 & 43 & 49 & 43 & 43 & 49 & 49 \\
Step size (ST) & 12 & 9 & 12 & 9 & 9 & 12 & 9 & 12 \\
Strain calculation window (SW) & 3 & 3 & 3 & 3 & 3 & 3 & 3 & 3 \\
VSG = ((SW-1)ST)+1 & 25 & 19 & 25 & 19 & 19 & 25 & 19 & 25 \\
Spatial resolution = ((SW-1)ST)+SS & 59 & 61 & 67 & 67 & 61 & 67 & 67 & 73 \\
Standard deviation & 1.12 & 1.07 & 0.94 & 0.84 & 0.80 & 0.62 & 0.55 & 0.45 \\
\hline
\end{tabular}

Table 2. Selected results of the virtual strain gauge (VSG) study under stress intensity factors of 20 and $30 \mathrm{MPa} \sqrt{\mathrm{m}}$, with four most promising parameter sets included. 


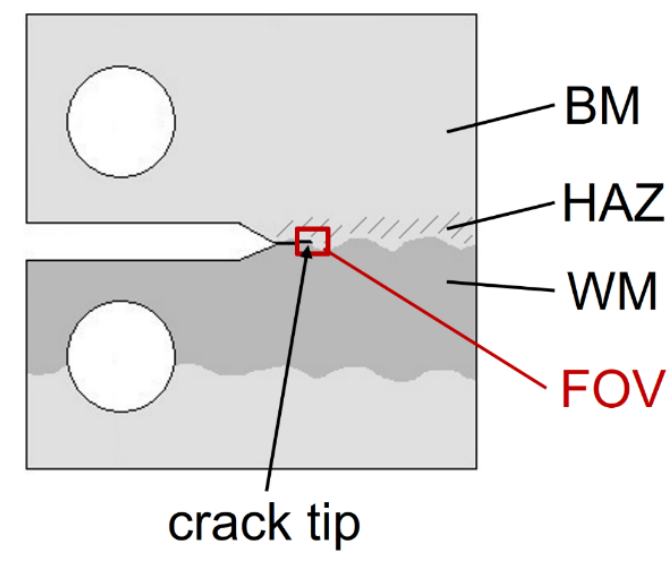

Fig. 1. A schematic of a welded CT specimen used for the DIC analysis, showing base metal (BM), weld metal (WM) and heat affected zone (HAZ) near the weld line. The field of view (FOV=2456 $x$ 2058 pixels $^{2} / 1330 \times 1120 \mu^{2}$ ) is also indicated.

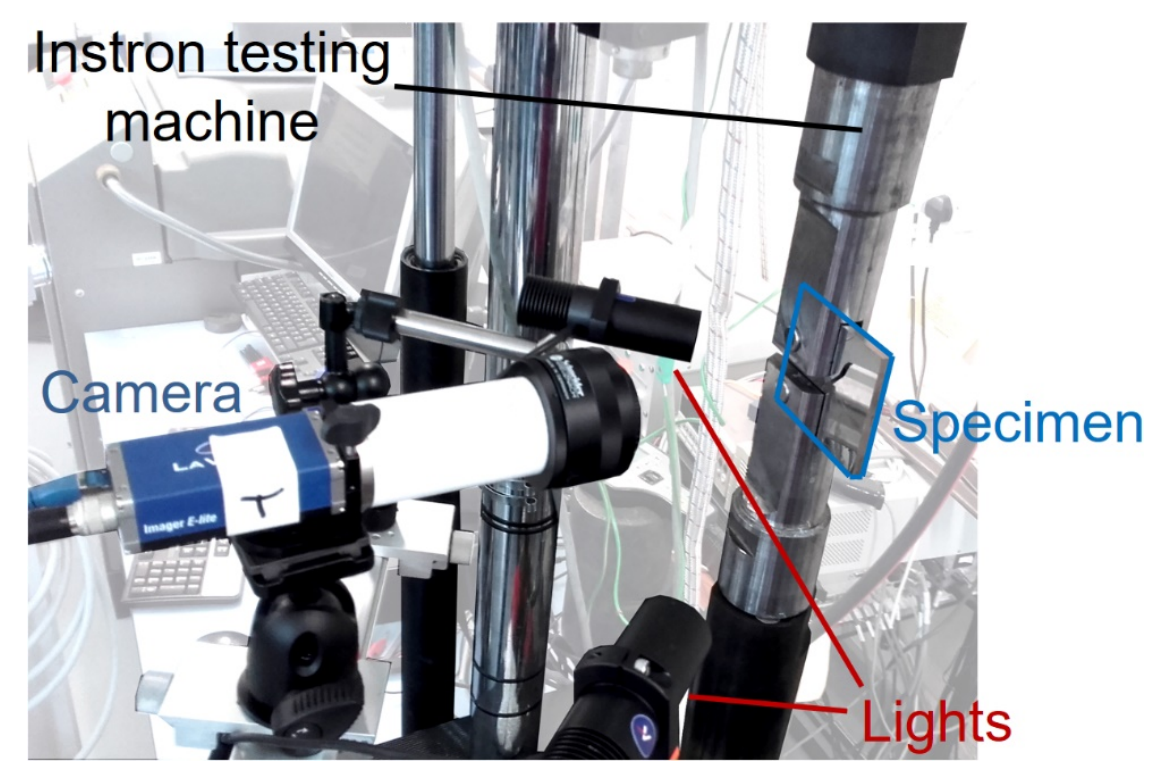

Fig. 2. The experimental setup for in situ DIC imaging of a CT specimen on an Instron servo-hydraulic testing rig. 
a)

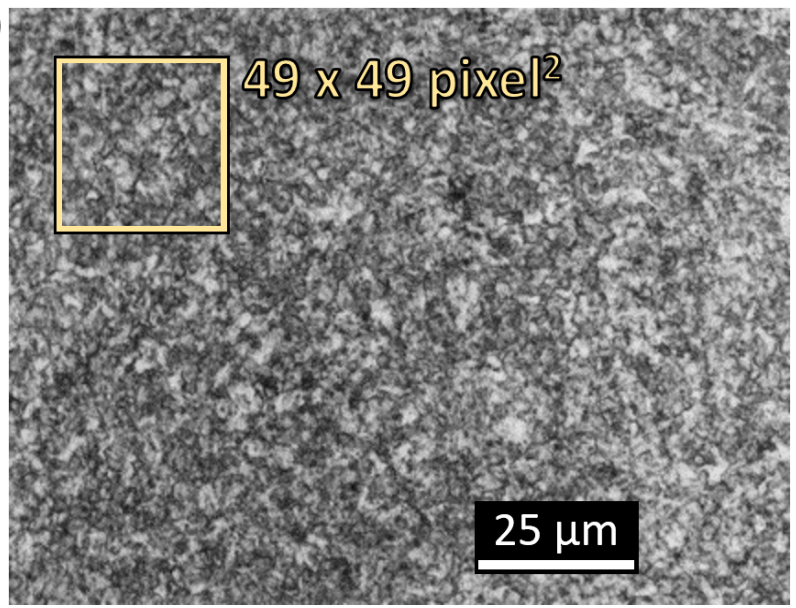

b)

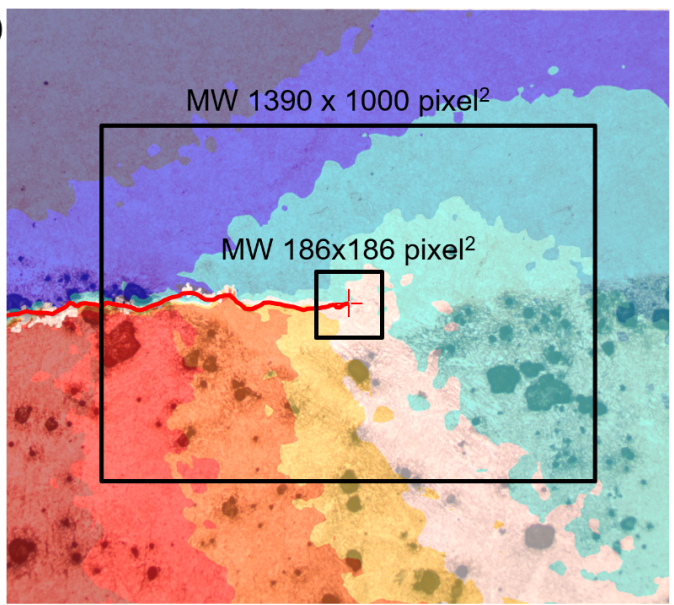

Fig. 3. a) Micrograph of an etched specimen surface in the area of interest near the crack tip with a typical subset illustrated. The spatial resolution achieved using the information of the surface texture is $0.54 \mu \mathrm{m} /$ pixel. b) The displacement field superimposed on a micrograph with a sketch of two sizes of measurement windows (MW) positioned around the crack tip. 

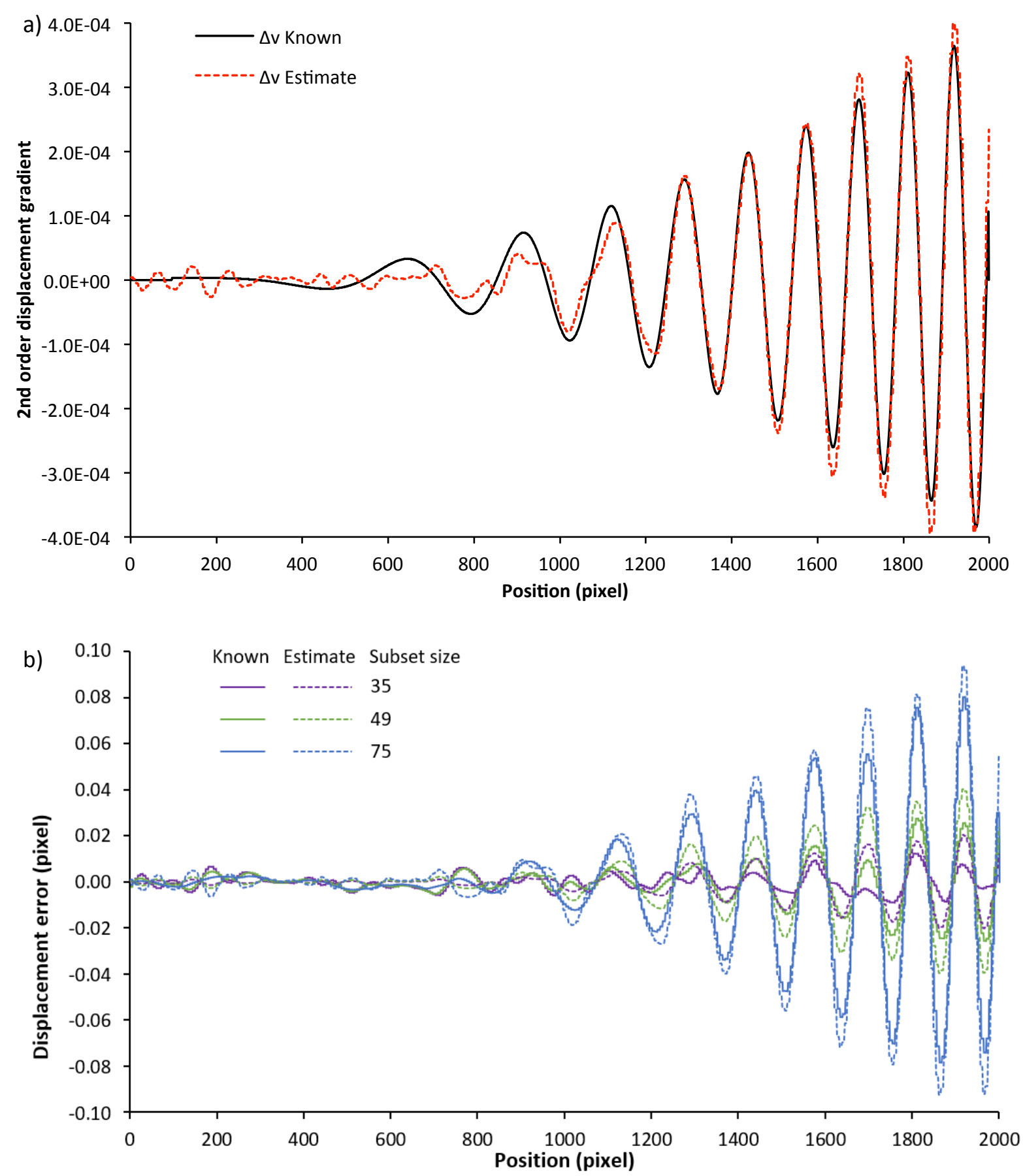

Fig. 4. a) A comparison of the known second order displacement gradient $\Delta v$ (black line, data from DIC challenge sample $14 \mathrm{~L}^{[51]}$ ) with the estimated gradient (red line, from Eq 2 , using subset sizes 35 and 75 pixels); b) A comparison of the actual displacement bias (solid lines, the difference between the known displacements in sample $14 L 5^{[51]}$ and the DIC generated displacements from the same sample) with the approximated errors (dashed lines, from Eq 1) for selected subset sizes of $35,49,75$ pixels. 

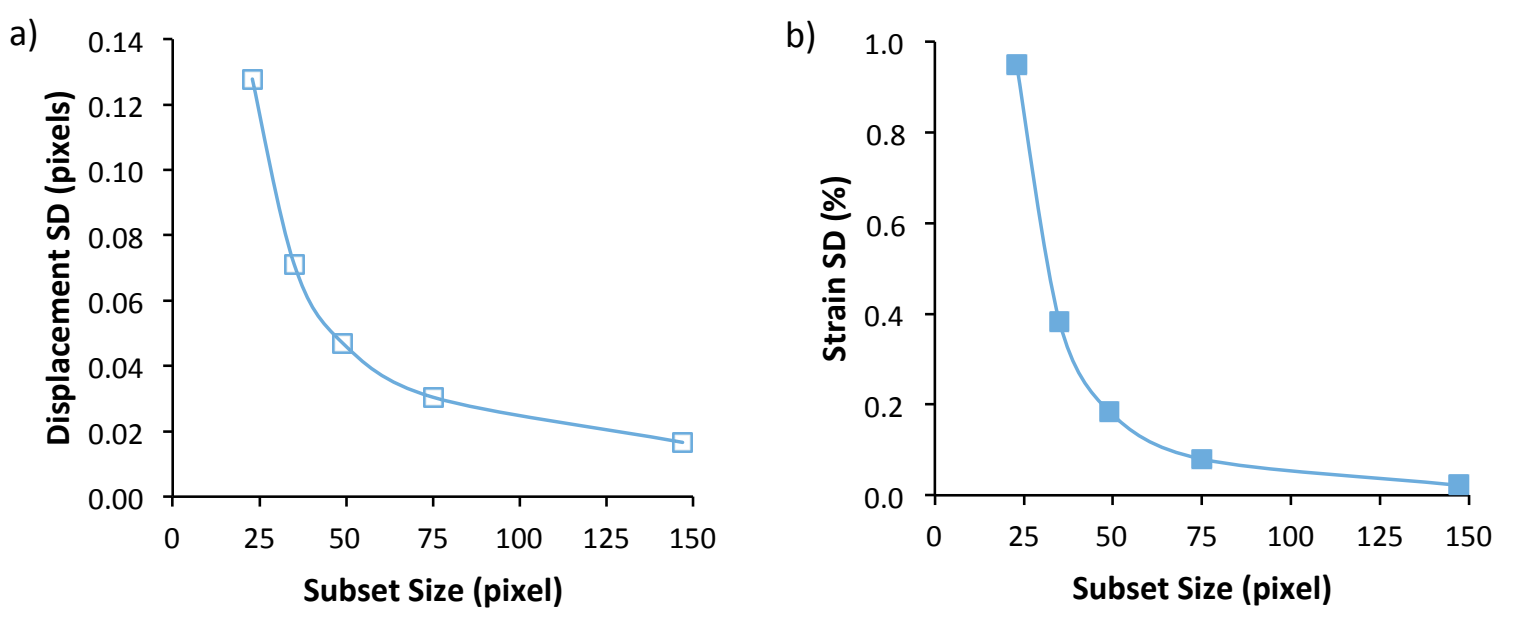

Fig. 5. The standard deviation (SD) of (a) displacement and (b) strain in y-direction at zero load (noise floor) as a function of subset size. The errors are reduced significantly when the subset size is above 50 pixels. RMSE is identical to SD, and is omitted here for conciseness. The step size is taken as 6 pixels. 


\section{Random}

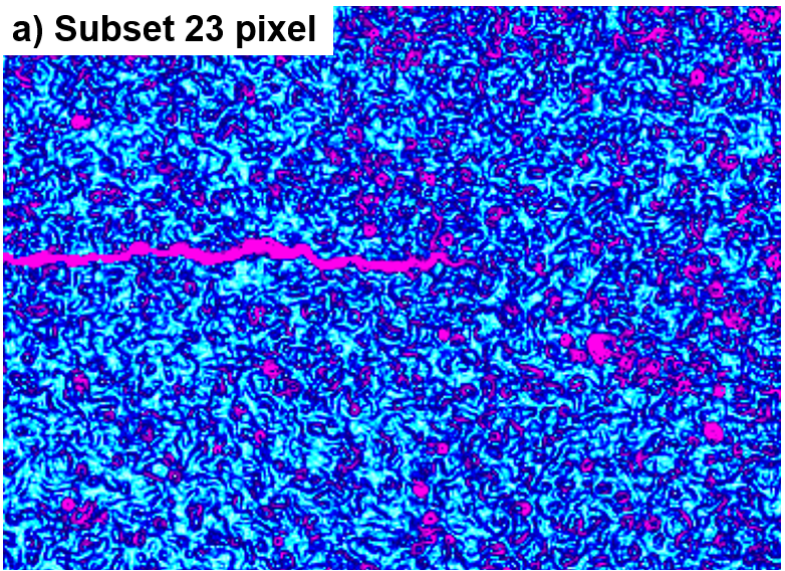

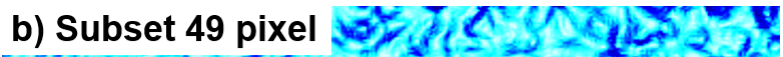

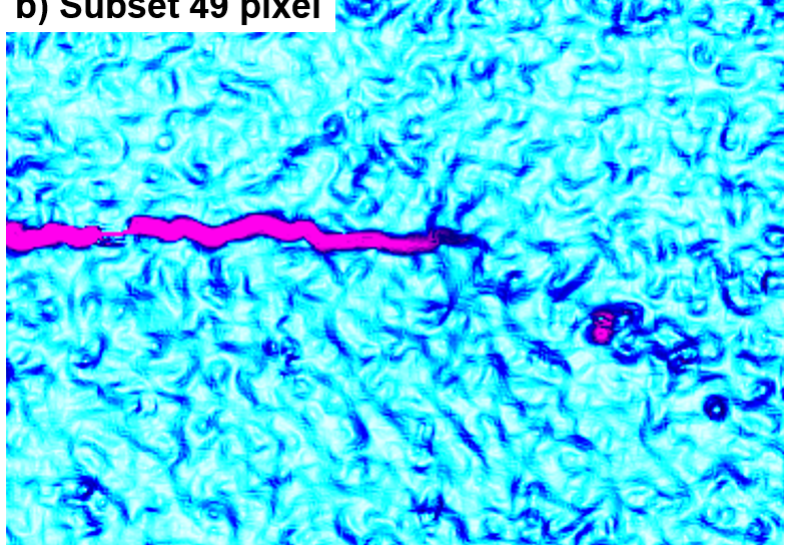

\section{c) Subset 75 pixel}
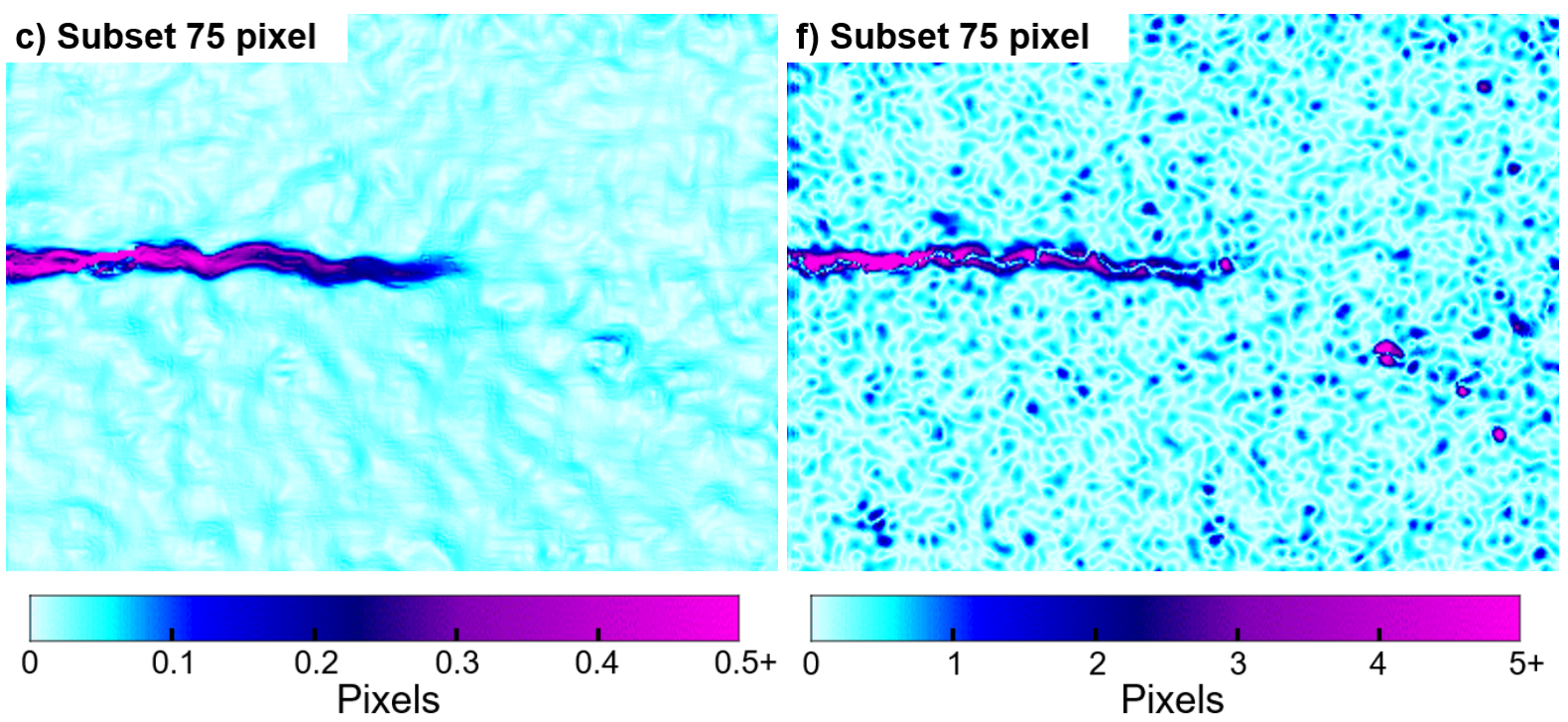

d) Subset 23 pixel

e) Subset 49 pixel

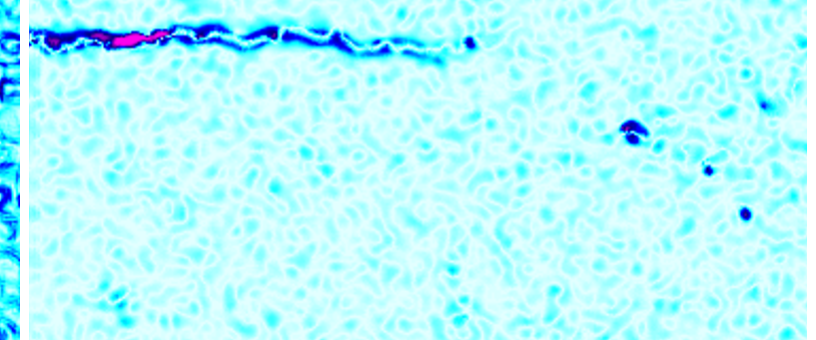

Fig. 6. The full-field results of displacement errors of the area correlated $\left(1250 \times 900 \mu \mathrm{m}^{2}\right.$ or $2320 \mathrm{x}$ 1670 pixels $^{2}$ ) under $\mathrm{K}=20 \mathrm{MPa} \sqrt{\mathrm{m}}$ : Random errors (left column) and systematic errors (right column) for the selected subset sizes. 

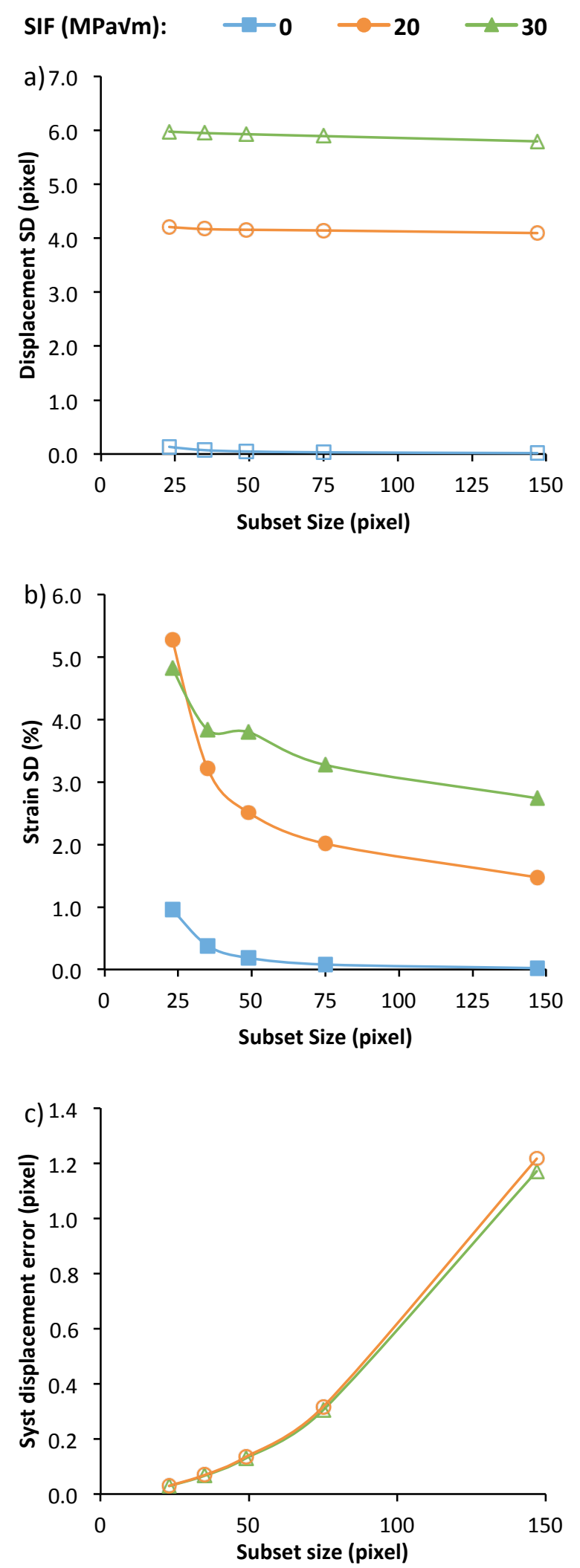

Fig. 7. The effects of the subset size on the random errors of (a) displacement and (b) strain in $y$ direction for the three load cases $(n=15)$. (c) The effects of the subset size on the estimated systematic displacement errors in $y$-direction for the loaded cases $(n=15)$. The step size is taken as 6 pixels. 
SIF (MPaVm): $\quad-0 \quad-20 \div 30$
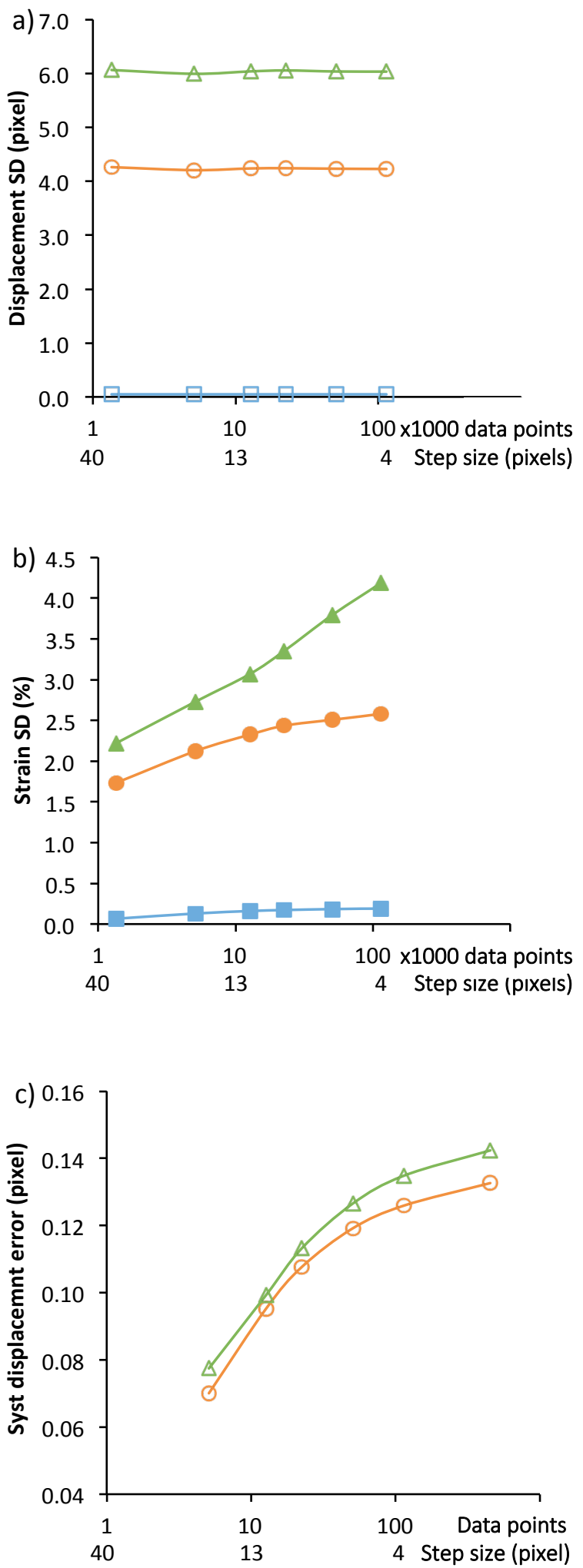

Fig. 8. The effects of step size on the random errors of (a) displacement and (b) strain in y-direction; (c) the effects of step size on the estimated systematic displacement errors in y-direction; presented as function of the step size and the number of data points in the measurement window. The subset size is fixed at $49 \times 49$ pixels $^{2}$. 

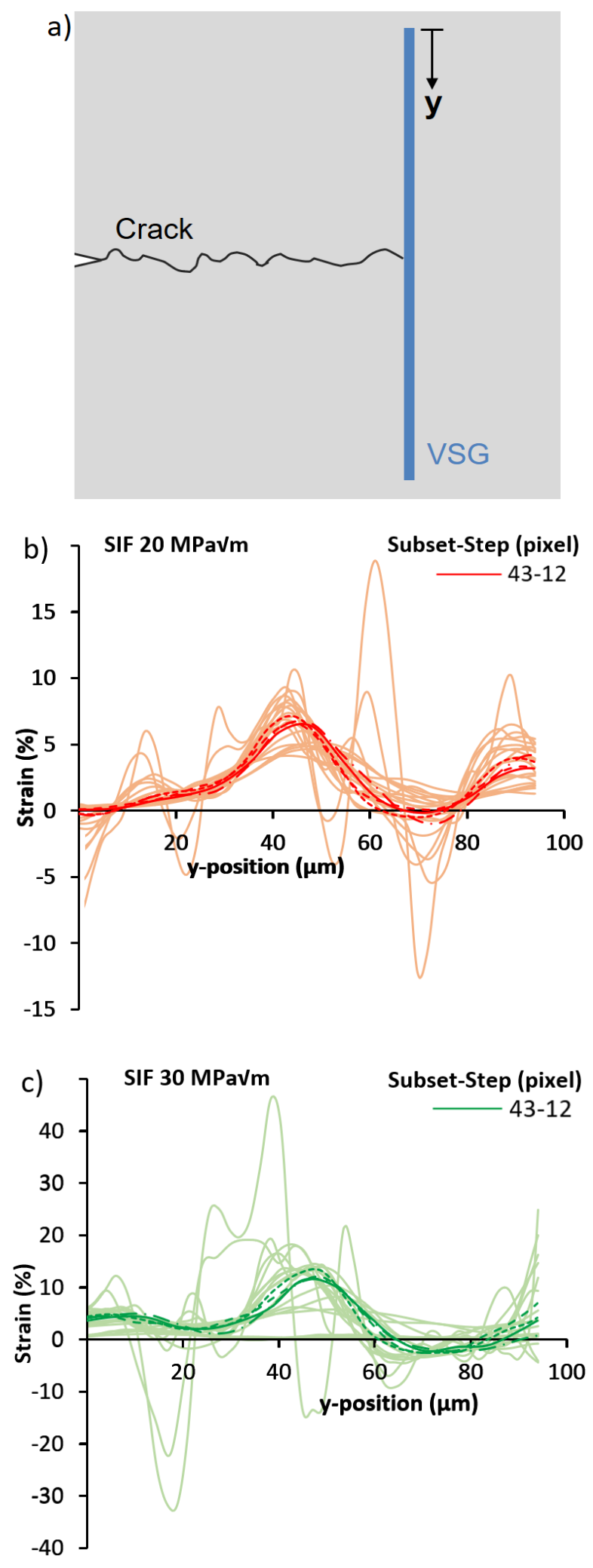

Fig. 9. (a) An illustration of a Virtual Strain Gauge (VSG) placed along a line (94 $\mu \mathrm{m})$ perpendicular to the crack plane centred at the crack tip. The normal strains obtained under a stress intensity factor of (b) $20 \mathrm{MPa} \sqrt{\mathrm{m}}$ and (c) $30 \mathrm{MPa} \sqrt{\mathrm{m}}$ are presented. Light-weighted lines show the results from all combinations of subset sizes $(23,35,43,49,61,75$ pixels) and step sizes $(4,6,9,12,19$ pixels), with the most promising four sets (subset size-step size $=35-12 ; 43-9 ; 43-12$ and $49-9$ ) highlighted. The "best" parameter set seems to be 43-12, shown as a solid line. The details are given in Table 2. 
SIF (MPaVm): $\quad-0 \div 20-30$
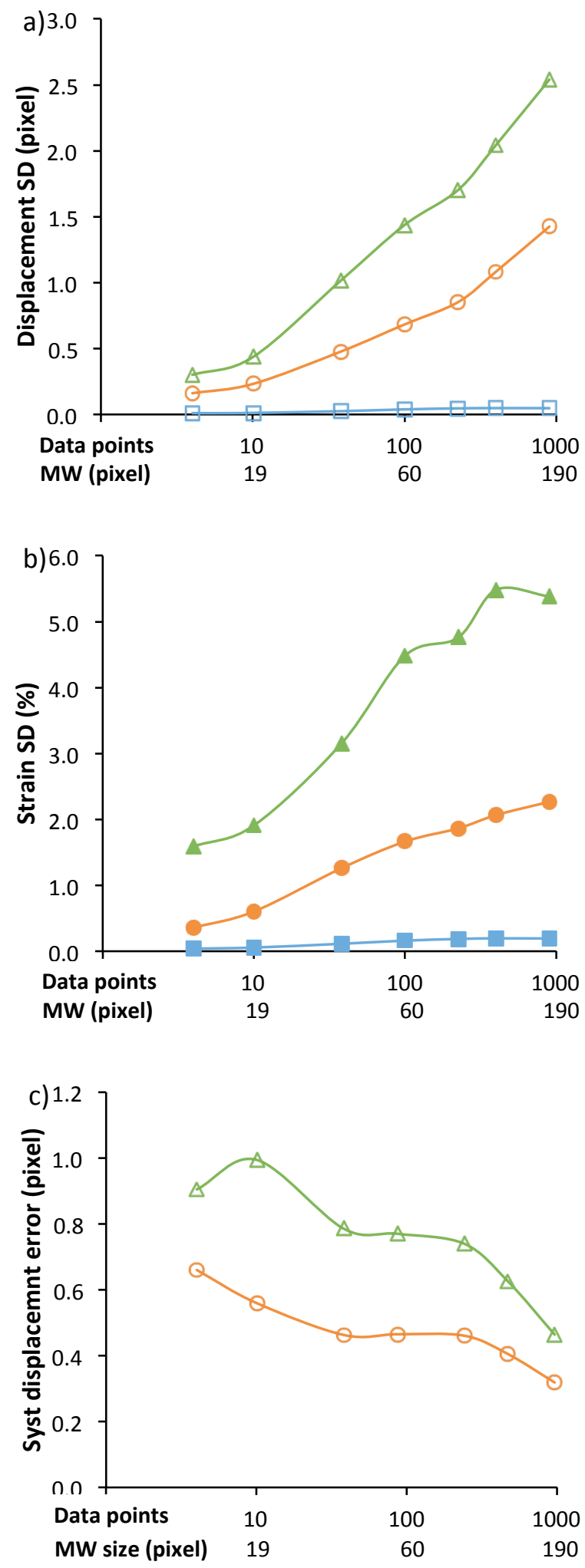

Fig. 10. The effects of the size of measurement window (MW) on the random errors of (a) displacement and (b) strain in y-direction; (c) the effects of the size of MW on the estimated systematic displacement errors in y-direction. A constant subset size of $49 \times 49$ pixels ${ }^{2}$ and a step size of 6 pixels are used. 